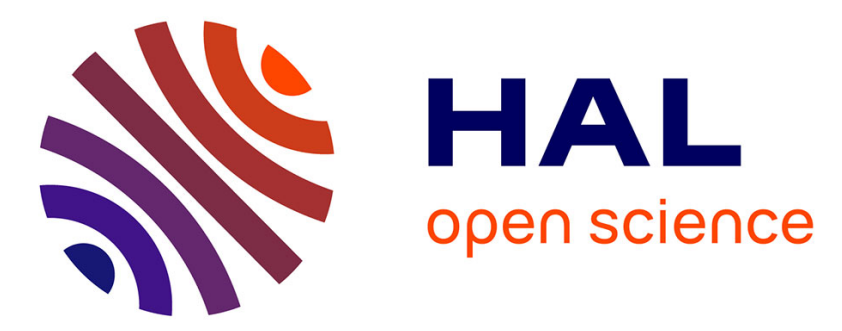

\title{
Diamond Particle Detectors for High Energy Physics
}

W. Trischuk, M. Artuso, F. Bachmair, L. Bäni, M. Bartosik, V. Bellini, V.

Belyaev, B. Bentele, E. Berdermann, P. Bergonzo, et al.

\section{To cite this version:}

W. Trischuk, M. Artuso, F. Bachmair, L. Bäni, M. Bartosik, et al.. Diamond Particle Detectors for High Energy Physics. Nuclear and Particle Physics Proceedings, 2016, 273-275, pp.1023-1028. 10.1016/j.nuclphysbps.2015.09.160 . in2p3-01343943

\section{HAL Id: in2p3-01343943 \\ https://hal.in2p3.fr/in2p3-01343943}

Submitted on 19 Jul 2019

HAL is a multi-disciplinary open access archive for the deposit and dissemination of scientific research documents, whether they are published or not. The documents may come from teaching and research institutions in France or abroad, or from public or private research centers.
L'archive ouverte pluridisciplinaire HAL, est destinée au dépôt et à la diffusion de documents scientifiques de niveau recherche, publiés ou non, émanant des établissements d'enseignement et de recherche français ou étrangers, des laboratoires publics ou privés. 


\title{
Diamond Particle Detectors for High Energy Physics
}

\author{
William Trischuk \\ Department of Physics, University of Toronto, 60 Saint George St., Toronto, Ontario, Canada
}

On behalf of the RD42 Collaboration - see Appendix A

\begin{abstract}
Diamond devices have now become ubiquitous in the LHC experiments, finding applications in beam background monitoring and luminosity measuring systems. This sensor material is now maturing to the point that the large pads in existing diamond detectors are being replaced by highly granular tracking devices, in both pixel and strip configurations, for detector systems that will be used in Run II at the LHC and beyond. The RD42 collaboration has continued to seek out additional diamond manufacturers and quantify the limits of the radiation tolerance of this material. The ATLAS experiment has recently installed, and is now commissioning a fully-fledged pixel tracking detector system based on diamond sensors. Finally, RD42 has recently demonstrated the viability of 3D biased diamond sensors that can be operated at very low voltages with full charge collection. These proceedings describe all of these advances.
\end{abstract}

Keywords: Particle detectors, radiation tolerance, precision tracking, high energy physics

\section{CVD Diamond Material}

Diamond has a number of properties that make it an attractive alternative to silicon as a sensor material for solid state particle detection applications. Its large band-gap and consequent small leakage current have allowed the production of position sensitive devices by simply patterning electrodes on the surface, as opposed to implanting diode junctions in silicon. Furthermore the large band-gap and strong atomic bonds make diamond material much less susceptible to the radiation doses typically found in modern high energy collider physics experiments. Its low dielectric constant means that for a given sensor area, the load capacitance on a readout amplifier will be half that of a similar shaped silicon detection element. Finally the high band-gap allows the saturation the drift field producing prompt signals, with rise-times below one nanosecond. The fast signals in diamond detectors have already been exploited to provide single turn feedback on beam conditions near experimental interaction regions and to make bunch-by-bunch measurements of the collider luminosity. All four LHC experiments have diamond beam monitors, based on single large pad electrodes, currently in operation and their focus is now turning to the use of diamond sensors with segmented readout geometry for use in charged particle tracking applications.

The RD42 collaboration [1] has been developing particle detector prototypes based on Chemical Vapour Deposited (CVD) diamond sensor material for two decades. Over the last few years our main focus has been building particle detector prototypes and promoting their transition to fully-fledged detector systems in the LHC experiments. It is now possible to routinely grow polycrystalline CVD diamond wafers $12-15 \mathrm{~cm}$ in diameter that produce most-probable signals approaching 10000 electrons from the passage of a minimum ionising particle. This is the result of a 10-year collaborative development effort between RD42 and Element6 [2] and a more recent two year, accelerated development cycle with II-VI Incorporated [3]. Figure 1 shows one of three wafers that have been produced by 


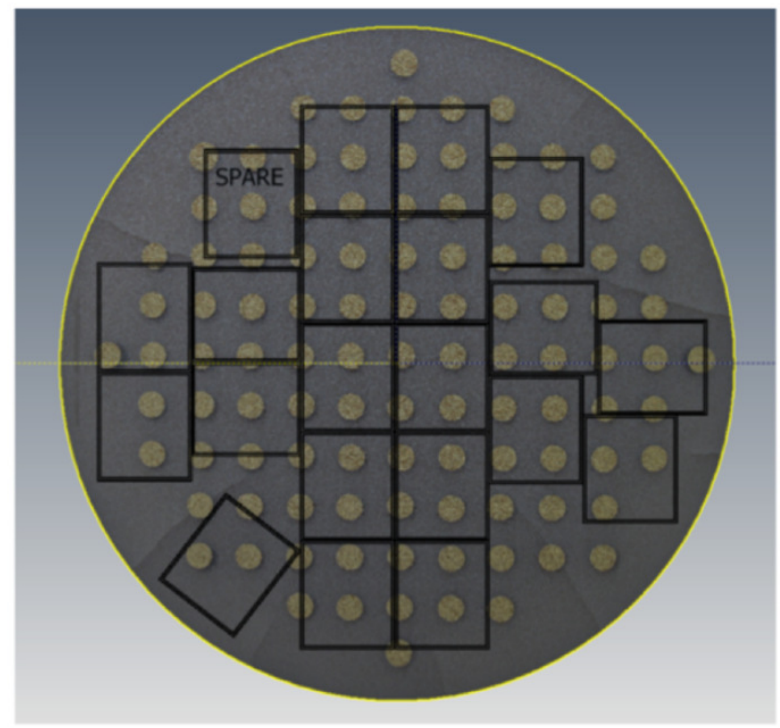

Figure 1: A14 $\mathrm{cm}$ diameter wafer, produced in 2013 by II-VI. The marked areas show where twenty one, potential, sensors for the ATLAS-DBM were cut from this wafer. Nine sensors from this company have been assembled into pixel modules that are installed in the ATLAS experiment.

II-VI, parts of which have been provided to members of RD42 to produce prototype position sensitive detectors for HEP applications. With two manufacturers we have even begun to see prices for this material come down. This diamond material has also been tested for radiation hardness over the last several years and shows acceptable degradation up to fluences of $10^{16}$ protons per $\mathrm{cm}^{2}$ - typical of the expected fluences in the inner layers of HL-LHC trackers. I will describe radiation tolerance measurements from RD42 on recent pCVD material, the construction of the ATLAS Diamond Beam Monitor and a novel diamond sensor geometry that uses $3 \mathrm{D}$ electrode structures that may result in even more radiation tolerant diamond sensors in the future.

\section{Radiation Tolerance of Diamond Sensors}

Over the last five years the RD42 collaboration has carried out an extensive program of diamond sensor irradiation and charged particle signal characterisation to quantify the radiation tolerance of the diamond material currently available to produce HEP detector systems. We have studied the effect of proton, pion and neutron irradiations. While charged pions dominate the flux at hadron colliders, protons (of various energies) are more readily available for controlled irradiations to fluences that will typically be seen over the lifetime of the LHC. We have measured the effect of $24 \mathrm{GeV} / \mathrm{c}, 700 \mathrm{MeV} / \mathrm{c}$,

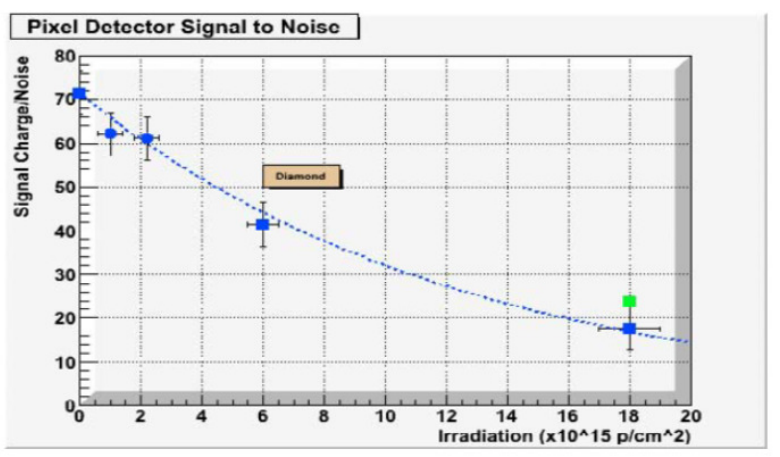

Figure 2: MIP signals measured in diamond strip trackers interpreted as signal-to-noise in a pixel device - using a 100 electron noise typical of FE-I4 single pixels. The green point, at the highest fluence, shows the signal when the electric field on the sensor is doubled to $2 \mathrm{~V} / \mu \mathrm{m}$ while all other points are measured at $1 \mathrm{~V} / \mu \mathrm{m}$.

$300 \mathrm{MeV} / \mathrm{c}$ and $24 \mathrm{MeV} / \mathrm{c}$ protons at fluences comparable to those expected at the full lifetime of the HL-LHC. Figure 2 shows the effect of $24 \mathrm{GeV} / \mathrm{c}$ proton fluences up to $1.8 \times 10^{16}$. The surviving signal is measured, after the irradiation, with single MIPs in a $100 \mathrm{GeV}$ proton testbeam at CERN. In this figure the signal observed is converted to $\mathrm{S} / \mathrm{N}$ using the typical noise (see below) seen in diamond pixel prototypes - 100 electrons for pixel pads of $50 \times 250 \mu \mathrm{m}^{2}$ and the FE-I4 readout chip. While it is still a challenge to reliably trigger the readout of a pixel chip with a threshold of less than 2000 electrons, such as would be needed for high efficiency operation at the highest fluences shown here, advances in chip design that are being made to efficiently measure signals in heavily irradiated silicon sensors should make this possible.

RD42 has performed similar measurements after exposures to lower fluences of lower energy protons and pions. We are building a picture of radiation tolerance in diamond, similar to the NIEL model for silicon sensors. Preliminary measurements show that damage scaling to lower energies and other particle species result in less damage in diamond than would be expected from a direct translation of NIEL from silicon. While this is encouraging, work is underway to quantify these results and build models that explains the differences.

\section{A Diamond Pixel Tracker: The ATLAS DBM}

As the luminosity increases, current beam monitoring systems will saturate as all modules are hit with at least one MIP in every collision. For example, the current ATLAS Beam Conditions Monitors will begin to saturate when the instantaneous luminosity reaches 


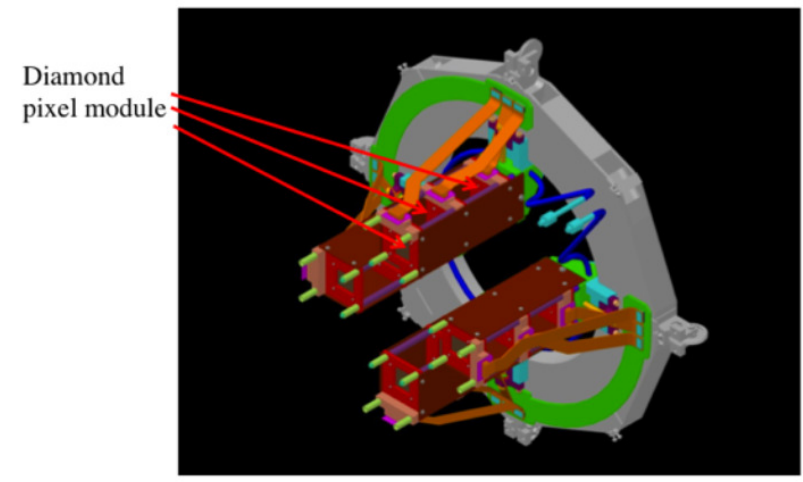

Figure 3: A CAD model of one half of the ATLAS DBM, that shows four telescopes that are each composed of three diamond pixel modules.

$\mathcal{L} \approx 10^{34} / \mathrm{cm}^{2} / \mathrm{s}$. The Diamond Beam Monitor (DBM) [4] has been designed with much better segmentation, using a pixel pattern of almost $27 k$ individual readout cells, overcomes this limitation. This section describes more of the design and construction of the ATLAS DBM.

In the DBM each diamond sensor has dimensions of $18 \mathrm{~mm} \times 21 \mathrm{~mm} \times 500 \mu \mathrm{m}$. One face of the diamond sensor is a conducting backplane to supply the high voltage bias while the other side is metalised with a pixelated pattern that can be bump bonded to the pixel electronics, a front-end I4, designed for the ATLAS Insertable Barrel Layer [5] (IBL). The individual FEI4 pixel size is $50 \times 250 \mu \mathrm{m}^{2}$ arranged into 80 columns ( $250 \mu \mathrm{m}$ wide) each having 336 rows ( $50 \mu \mathrm{m}$ high). The construction of a diamond pixel module is identical to that of a silicon pixel module, the only difference being the eventual deployment of these modules into larger detector structures for installation. The DBM consists of four telescopes on each side of ATLAS. An engineering model for one side is shown in Fig 3. Each telescope points towards the centre of ATLAS (to the right in this sketch) and consists of three diamond modules. The telescopes are mounted at $|z|=0.93 \mathrm{~m}$ and cover rapidities of $3.2<|\eta|<3.5$. This location was the closest to the IP and beamline that was available and was occupied to maximise the DBM acceptance given the fixed size of the individual modules. Sensors from E6 and II-VI were used in the assembly of the DBM modules.

The diamond pixel modules were bump bonded by IZM [6]. About half of the diamond modules produced required rework to achieve acceptable bond connectivity. Figure 4 shows the hit maps for four of the diamond

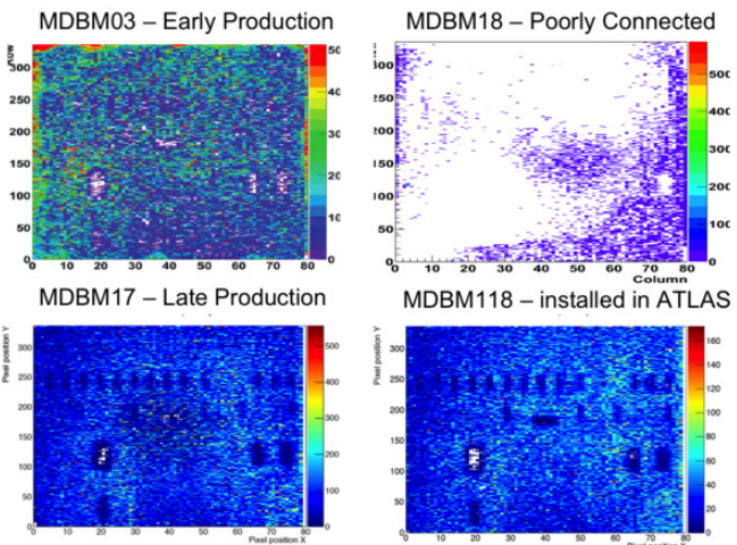

Figure 4: Four occupancy scans that were taken as part of the ATLAS DBM module quality assurance testing. One module MDBM18, showed very poor connectivity. It was disassembled, cleaned and rebonded, resulting in MDBM118 (bottom right) that showed fully connectivity and is now installed in the ATLAS-DBM, along with the other two modules shown here.

pixel modules assembled. These hit maps were obtained with a two dimensional scan of $2 \mathrm{MeV}$ electrons from a ${ }^{90} \mathrm{Sr}$ source. Three of these modules show good connectivity - a uniform distribution of hits in all pixels. The hit map on the top right (MDBM18) was not uniform. Similarly poor connectivity was seen on a number of assemblies during the middle of the DBM module production. The poorly connected modules were disassembled, their pixel patterns were cleaned and a second bonding was attempted. The module on the bottom right (MDBM118) is the result of this re-working for the same diamond sensor. As a result of this work we (and IZM) have learned a great deal about the subtleties of bump-bonding diamond sensors. The differential thermal expansion between the silicon readout chip and the diamond sensor is just one potential issue that complicates the bonding process. However we explored a large number of other process variations with IZM over the course of this project and now have a viable bonding procedure for diamond sensors. The necessity of reworking was the largest hurdle overcome in moving from a handful of diamond pixel module prototypes to a full detector system in ATLAS.

During the course of the DBM module quality assurance process we attempted to tune the individual pixel readout thresholds to the lowest practical values possible, in order to achieve the highest single MIP hit efficiencies, while still maintaining acceptably low noise rates. In practice, the FE-I4 chip can run reliably - with an acceptable number of noisy pixels - down to thresh- 


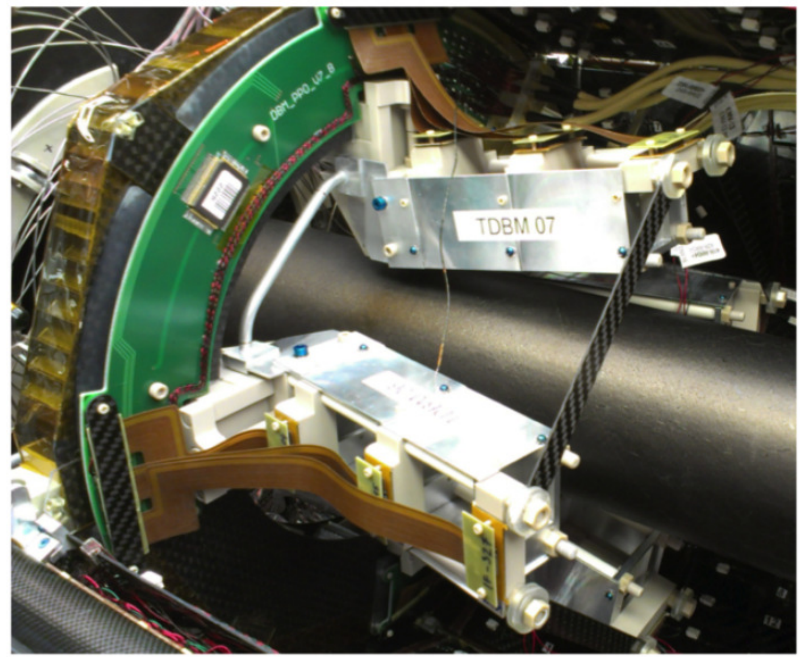

Figure 5: A photo of the ATLAS Diamond Beam Monitor telescopes installed on the pixel support frame, prior to re-installation of the whole package in ATLAS in the late fall of 2013.

olds of about 1000 electrons. However, in the course of studying this in our diamond modules a number of non-linearities and spatial variations (across the relatively large FE-I4 chip) in the thresholds were identified and we are developing tuning algorithms to achieve the lowest possible, uniform, threshold distribution for DBM module operation. While this is critical to the operation of diamond pixel modules - due to their lower initial MIP signal - it will be equally important for the ultimate operation of the IBL silicon modules, after they have received doses approaching $10^{15} / \mathrm{cm}^{2}$ towards the end of their lifetime. The DBM is a trail-blazer for the operation of irradiated silicon pixel detector systems.

Figure 5 shows two telescopes on one side of the DBM installed on the ATLAS pixel support frame. The full pixel detector, including the DBM has now been returned to the ATLAS experimental hall and reconnected to its services. All eight DBM telescopes are fully functional and now being commissioned/tuned awaiting first beam in 2015 .

\section{3D Diamond Sensors}

Recently the RD42 collaboration has begun to explore the possibility of producing 3-dimensional biasing electrode structures through the diamond sensor material. Inspired by similar work in silicon [7] these electrodes, with separations as small as $25-50 \mu \mathrm{m}$, can produce much larger, lateral, electric fields in the diamond, with much lower applied voltages. Figure 6 shows a photograph of the first functional 3D diamond prototype. In this device several areas were instrumented, some with just readout strips and others (near the top of the photo) with active $3 \mathrm{D}$ bias electrodes. The 3D bias columns are produced with a high power laser, that produces conducting graphitic carbon pillars through the diamond after relatively short (10s of seconds) exposures at each location. These conductive pillars are then biased (alternately to HV and ground) by a series of inter-digitated bias networks on the back fo the diamond sensor.

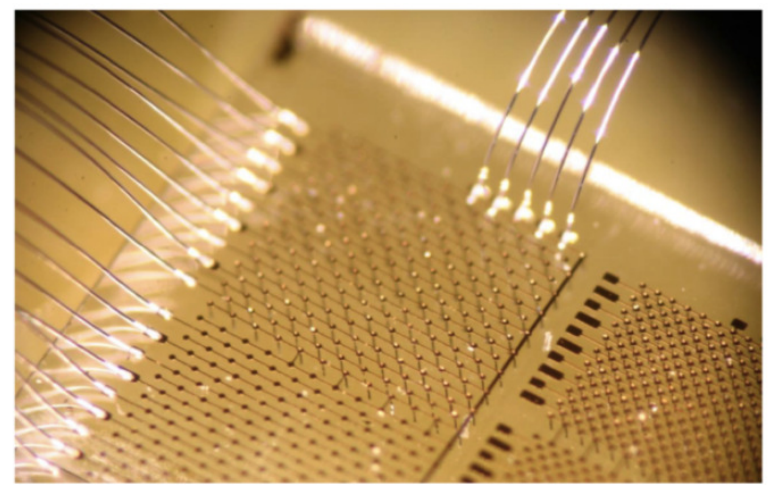

Figure 6: A photo of the first 3D diamond sensor produced and tested in a beam by RD42. The top left corner of this device includes 3D biasing electrodes and crossed readout strips that allow MIP pulseheights to be measured with a standard VLSI charged sensitive amplifiers in a testbeam. Below (and to the left) of that region are traditional $2 \mathrm{D}$ readout strips (and a corresponding, biased backplane). This allows an in-situ comparison of the signal collected for these two bias configurations. Other areas of the diamond (to the bottom right) were used to test the bias column generation parameters in the diamond material but were not readout in the testbeam.

The MIP signal properties of this device were tested at CERN in late 2012. High energy protons were tracked with a silicon reference telescope and the resulting pulse-heights in the 3D diamond prototype were recorded on strips that spanned 12 bias electrodes. Figure 7 shows the average pulse height recorded for a bias of $25 \mathrm{~V}$ as a function of lateral position across the sensor. The lateral position in this figure is determined from the external, reference, telescope and not the 3D diamond device. One sees a number of ares of lower pulseheight (the blue regions) where we have independent evidence that one (or more) of the biasing electrodes were not connected to the biasing network. However a relatively large, contiguous area of this prototype shows good response and quite uniform charge collection (the green/yellow areas).

We have studied the pulse height distribution for the $6 \times 6$ cell area in the middle right of the sensor, where 


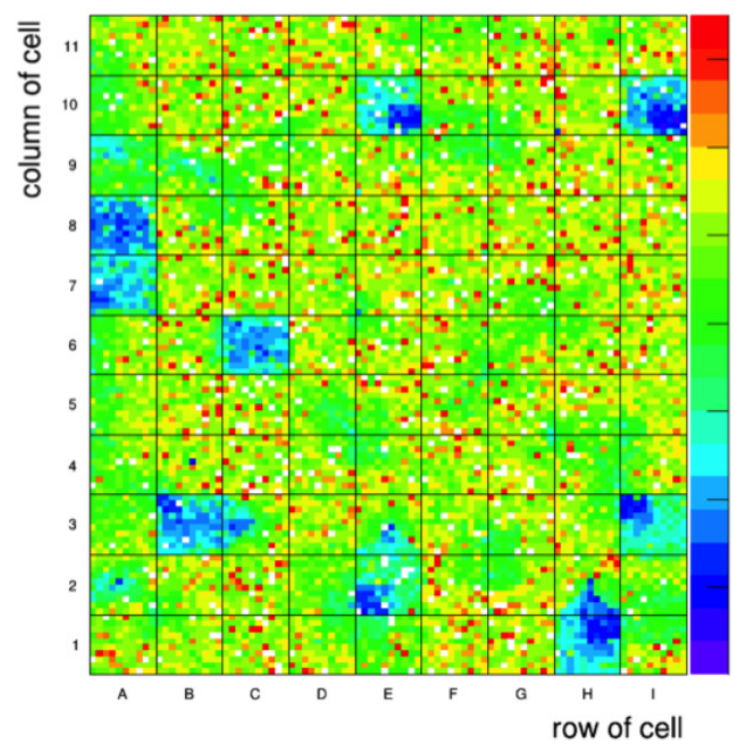

Figure 7: The average pulse height recorded in the 3D diamond prototype as function of lateral position across the sensor face. While a number of areas show lower than average pulse-height, large areas show uniform charge collection.

we had previously determined that no bias electrodes were disconnected from their, respective, biasing grids. Figure 8 shows the 3D pulse-height distribution (in blue) overlaid with the pulse height distribution from the neighbouring conventional strip tracker. One sees very good agreement between the two pulse-height distributions. The remarkable achievement here is that the conventional tracker requires a $500 \mathrm{~V}$ bias (across the $500 \mu \mathrm{m}$ thick sensor) while the 3D device is only biased with $25 \mathrm{~V}$. Encouraged by these initial results additional work is now underway to make more reliable and larger area 3D electrode structures in diamond and to explore the intrinsic position resolution of such $3 \mathrm{D}$ sensors.

\section{Summary}

Diamond sensors are finding widespread applications in high energy physics. Their radiation tolerance, fast signal formation time and dimensional stability have made them and ideal for use as beam monitors and luminosity detectors during Run I at the LHC. They are now being prepared for a variety of position sensitive detector applications very near the interaction region of the LHC experiments where high particle fluxes and radiation fluences discourage the use of other technologies. The ATLAS experiment has installed a set of pixel telescopes that use diamond sensors and is preparing to use

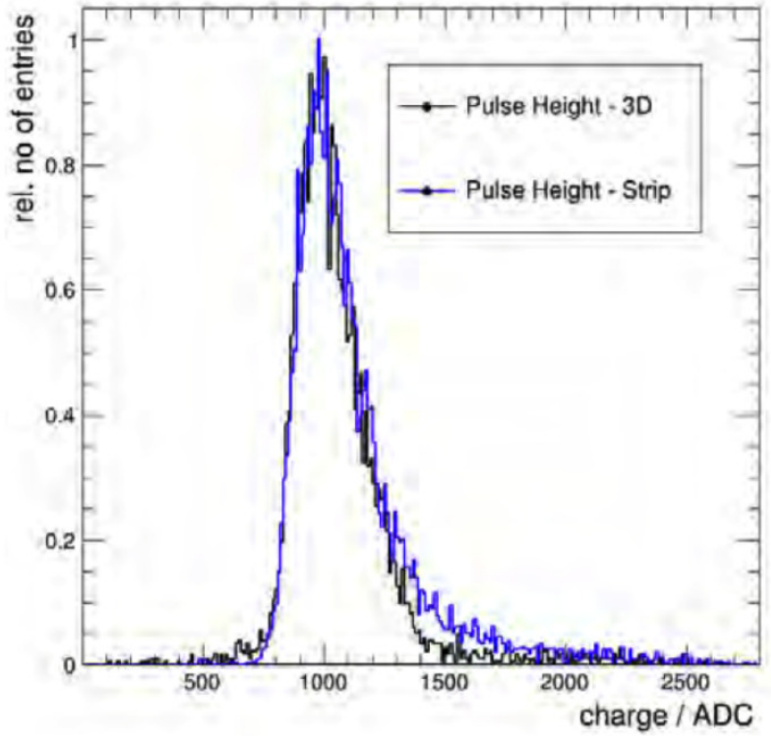

Figure 8: A direct comparison of pulse heights from two regions of the $3 \mathrm{D}$ prototype. The blue curve shows the result from the $3 \mathrm{D}$ electrode region, biased at $25 \mathrm{~V}$. The black curve shows the pulse heights from the traditional 2D diamond strip tracker, biased at $500 \mathrm{~V}$.

these augment their luminosity and background monitoring capabilities in run II. CMS and LHCb are exploring diamond sensors as options for their future pixel detector upgrades. The RD42 collaboration has also begun to explore the possibility of biasing diamond sensors with $3 \mathrm{D}$ electrode structures, potentially providing a significant reduction in the sensor bias voltages necessary to achieve full charge collection in these devices.

\section{References}

[1] M. Artuso et al. (The RD42 Collaboration), "RD42 Status Report: Development of Diamond Tracking Detectors for High Luminosity Experiments at the LHC", CERN/LHCC 2008-005. See also: http://indico.cern.ch/event/319702/ for the most recent update.

[2] Element Six Technologies, Global Innovation Centre, Fermi Ave., Harwell, OX11 0QR, United Kingdom.

[3] II-VI Incorporated, 375 Saxonburg Blvd., Saxonburg, PA, 16056-9499, USA.

[4] H.Kagan, M.Mikuz, W.Trischuk, "The ATLAS Diamond Beam Monitor TDR", ATLAS Document ATU-DBM-001, 09/14/2011.

[5] J.Albert et al. (The ATLAS-IBL Collaboration), "Prototype ATLAS IBL Modules using the FE-I4A Front-End Readout Chip", arXiv:1209.1906 [physics.ins-det].

[6] Fraunhofer Institute Zuverlassigkeit Mikrointegration, GustaveMeyer-Alle 25, D-13355, Berlin, Germany.

[7] S. Parker, C. Kenney and J. Segal "3D, a new architecture for solid state radiation detectors", Nucl. Instrum. Meth. A395 (1997). 


\section{Appendix A. The RD42 Collaboration}

M. Artuso ${ }^{27}$, F. Bachmair ${ }^{32}$, L. Bäni ${ }^{32}$, M. Bartosik ${ }^{5}$, V. Bellini ${ }^{4}$, V. Belyaev ${ }^{21}$, B. Bentele ${ }^{2}$, E. Berdermann ${ }^{1}$, P. Bergonzo ${ }^{19}$, A. Bes ${ }^{12}$, J-M. Brom ${ }^{14}$, M. Bruzzi ${ }^{8}$, M. Cerv ${ }^{5}$, C. Chau ${ }^{31}$, G. Chiodini ${ }^{18}$, D. Chren ${ }^{6}$, V. Cindro ${ }^{17}$, G. Claus ${ }^{14}$, J. Collot ${ }^{12}$, S. Costa ${ }^{4}$, J. Cumalat ${ }^{2}$, A. Dabrowski ${ }^{5}$, R. D'Alessandro ${ }^{9}$, W. de Boer ${ }^{16}$, B. Dehning ${ }^{5}$, D. Dobos ${ }^{5}$, W. Dulinski ${ }^{14}$, V. Eremin ${ }^{26}$, R. Eusebi ${ }^{29}$, G. Forcolin ${ }^{20}$, J. Forneris ${ }^{30}$, H. Frais-Kölbl ${ }^{7}$, K.K. Gan ${ }^{23}$, M. Gastal ${ }^{5}$, M. Goffe ${ }^{14}$, J. Goldstein ${ }^{3}$, A. Golubev ${ }^{15}$, L. Gonella ${ }^{1}$, A. Gorišek ${ }^{17}$, L. Graber ${ }^{11}$, E. Grigoriev ${ }^{15}$, J. Grosse-Knetter ${ }^{11}$, M. Guthoff ${ }^{5}$, I. Haughton ${ }^{20}$, D. Hidas ${ }^{25}$, D. Hits ${ }^{32}$, M. Hoeferkamp ${ }^{22}$, T. Hofmann ${ }^{5}$, J. Hosslet ${ }^{14}$, J-Y. Hostachy ${ }^{12}$, F. Hügging ${ }^{1}$, H. Jansen ${ }^{5}$, J. Janssen ${ }^{1}$, H. Kagan ${ }^{23, \diamond}$, K. Kanxheri ${ }^{24}$, G. Kasieczka ${ }^{32}$, R. Kass ${ }^{23}$, F. Kasse ${ }^{16}$, M. Kis ${ }^{1}$, G. Kramberger ${ }^{17}$, S. Kuleshov ${ }^{15}$, A. Lacoste ${ }^{12}$, S. Lagomarsino ${ }^{9}$, A. Lo Giudice ${ }^{30}$, C. Maazouzi ${ }^{14}$, I. Mandic ${ }^{17}$, C. Manfredotti ${ }^{30}$, C. Mathieu ${ }^{14}$, N. McFadden ${ }^{22}$, G. McGoldrick ${ }^{31}$, M. Menichelli ${ }^{24}$, M. Mikuž ${ }^{17}$, A. Morozzi ${ }^{24}$, J. Moss $^{23}$, R. Mountain ${ }^{27}$, S. Murphy ${ }^{20}$, A. Oh ${ }^{20}$, P. Olivero ${ }^{30}$, G. Parrini ${ }^{9}$, D. Passeri2 ${ }^{24}$, M. Pauluzzi ${ }^{24}$, H. Pernegger ${ }^{5}$, R. Perrino ${ }^{18}$, F. Picollo ${ }^{30}$, M. Pomorski ${ }^{19}$, R. Potenza ${ }^{4}$, A. Quadt ${ }^{11}$, A. Re ${ }^{30}$, G. Riley $^{28}$, S. Roe ${ }^{5}$, M. Sapinski ${ }^{5}$, M. Scaringella ${ }^{8}$, S. Schnetzer ${ }^{25}$, T. Schreiner ${ }^{7}$, S. Sciortino ${ }^{9}$, A. Scorzoni ${ }^{24}$, S. Seidel ${ }^{22}$, L. Servoli ${ }^{24}$, A. Sfyrla ${ }^{5}$, G. Shimchuk ${ }^{15}$, S. Smith ${ }^{23}$, B. Sopko ${ }^{6}$, V. Sopko ${ }^{6}$, S. Spagnolo ${ }^{18}$, S. Spanier ${ }^{28}$, K. Stenson ${ }^{2}$, R. Stone ${ }^{25}$, C. Sutera ${ }^{4}$, A. Taylor ${ }^{22}$, M. Traeger ${ }^{1}$, D. Tromson ${ }^{19}$, W. Trischuk ${ }^{31, \diamond}$, C. Tuve ${ }^{4}$, L. Uplegger ${ }^{10}$, J. Velthuis ${ }^{3}$, N. Venturi ${ }^{31}$, E. Vittone ${ }^{30}$, S. Wagner ${ }^{2}$, R. Wallny ${ }^{32}$, J.C. Wang ${ }^{27}$, P. Weilhammer ${ }^{5}$, J. Weingarten ${ }^{11}$, C. Weiss ${ }^{5}$, T. Wengler ${ }^{5}$, N. Wermes ${ }^{1}$, M. Yamouni ${ }^{12}$, M. Zavrtanik ${ }^{17}$

${ }^{1}$ Universität Bonn, Bonn, Germany, ${ }^{2}$ University of Colorado, Boulder, CO, USA, ${ }^{3}$ University of Bristol, Bristol, UK, ${ }^{4}$ INFN/University of Catania, Catania, Italy, ${ }^{5}$ CERN, Geneva, Switzerland, ${ }^{6}$ Czech Technical Univ., Prague, Czech Republic, ${ }^{7}$ FWT, Wiener Neustadt, Austria, ${ }^{8}$ INFN/University of Florence, Florence, Italy, ${ }^{9}$ Department of Energetics/INFN, Florence, Italy, ${ }^{10}$ FNAL, Batavia, USA, ${ }^{11}$ Universität Goettingen, Goettingen, Germany, ${ }^{12}$ LPSC-Grenoble, Grenoble, France, ${ }^{1} 3$ GSI, Darmstadt, Germany, ${ }^{14}$ IPHC, Strasbourg, France, ${ }^{15}$ ITEP, Moscow, Russia, ${ }^{16}$ Universität Karlsruhe, Karlsruhe, Germany, ${ }^{17}$ Jožef Stefan Institute, Ljubljana, Slovenia, ${ }^{18}$ INFN-Lecce, Lecce, Italy, ${ }^{19}$ CEA-LIST Technologies Avancees, Saclay, France, ${ }^{20}$ University of Manchester, Manchester, UK, ${ }^{21}$ MEPHI Institute, Moscow, Russia, ${ }^{22}$ University of New Mexico, Albuquerque, NM, USA, ${ }^{23}$ The Ohio State University, Columbus, OH, USA, ${ }^{24}$ INFN-Perugia, Perugia, Italy, ${ }^{25}$ Rutgers University, Piscataway, NJ, USA, ${ }^{26}$ Ioffe Institute, St. Petersburg, Russia, ${ }^{27}$ Syracuse University, Syracuse, NY, USA, ${ }^{28}$ University of Tennessee, Knoxville, TN, USA, ${ }^{29}$ Texas A\&M, College Park Station, TX, USA, ${ }^{30}$ University of Torino, Torino, Italy,

${ }^{31}$ University of Toronto, Toronto, ON, Canada, ${ }^{32}$ ETH Zürich, Zürich, Switzerland 